\title{
specificidad y dificultades de la restauración en jardinería
}

José Tito Rojo

Jardín Botánico de la Universidad de Granada

\section{Manuel Casares Porcel \\ Dpto. Biología Vegetal. Universidad de Granada}

Restauración de jardines, como de cualquier otro objeto artístico, ha habido siempre. La restauración de jardines como problema teórico es, sin embargo, reciente. La mayoría de los trabajos que tratan sobre ella son cercanos en el tiempo y todavía en nuestro país han sido escasos los acercamientos escritos al tema. El asunto no deja de ser peligroso cuando desde hace poco se multiplican en nuestro país las restauraciones de jardines históricos cuyos responsables carecen de instrumentos metodológicos de los que poder servirse. Peligro que aumenta pues, además de un modelo teórico de intervención, es precisa una adecuación a las concretas circunstancias de nuestros jardines históricos. Para dejar el panorama perfilado en su complejidad es necesario destacar en ese erial la aparición en los últimos años de trabajos españoles que abordan con seriedad la restauración de jardines y es justo destacar entre ellos los publicados por Carmen Añón, autora especialmente preocupada por el tema, que ha aportado notables textos teóricos (Añón, 1993) y reflexiones sobre restauraciones concretas abordadas por ella y su equipo (Añón, 1991, 1992). Sobre estos mismos aspectos tratan también algunos de nuestros propios trabajos (Tito Rojo, 1997; Casares Porcel y Tito Rojo, 1998, 1999). En las páginas que continúan presentamos nuestra visión sobre lo que consideramos el núcleo del problema, lo específico del jardín como objeto artístico realizado con material viviente y las dificultades para aplicarle los mismos criterios de intervención aceptados para los realizados con materiales inertes.

\section{La forma prístina y las diferencias de las restauraciones del jardín y de los edificios}

De la misma manera que la restauración arquitectónica fue en gran medida deudora de la restauración pictórica, la restauración llegó al jardín a través de la restauración de edificios. Durante mucho tiempo su práctica ha ido a remolque de las vicisitudes de ésta, su carácter de arquitectura vegetal ha sido predominante sobre su carácter de obra viviente, quedando del vegetal en los proyectos su forma y su volumen, acaso su textura y alguna cualidad física, su olor, su color, lo estrictamente arquitectónico.
Los problemas de conservación-mantenimiento de los jardines son cualitativamente diferentes a los de las obras construidas con materia dura. Mientras en los edificios el mantenimiento es un problema menor, en los jardines es una cuestión de tratamiento ineludible. En el jardín conservar es restaurar, el mantenimiento es una restauración, razón por la que, desde antiguo, en algunos tratados de jardinería así se contempla. Ya en la primera mitad del siglo XVIII, Dezallier d'Argenville, fijador teórico de las concepciones del jardín barroco francés, se adelantaba más de cien años a Viollet le Duc cuando afirmaba:

Quand on veut rajuster un vieux jardin sans le ruiner entièrement il faut alors en lever le plan exactement, \& examiner chaque piéce l'une après l'autre devant de la condamner. Ces ajustemens sont plus difficiles que d'inventer un dessein pour une place neuve, il gênent entrêmement le genie, \& l'obligent à bien des changemens avant que d'inventer un dessein qui s'ajuste à toutes les autres piéces plantées. Entrez tant que vous pourrez dans l'esprit de celui qui a donné le plan général du Jardin; accommodez-vous à ce qui est déja fait; corrigez le mauvais sans trop abattre; \& si vous y faites des piéces neuves, qu'elles ne soint pas si grandes, ni si magnifiques qu'elles effacent tout le reste. Les piéces simples s'accordent souvent mieux avec un vieux Jardin, que les morceaux riches \& extraordinaires. Cherchez plutôt à former un tout agréable, qu'à vous singulariser!.

El texto recoge las grandes líneas del restauro violletiano, la concepción de un plan original, la necesidad de ponerse en el lugar del primer diseñador, sin olvidar la llamada a la unidad del conjunto resultante y a la modestia, en la petición de que el nuevo diseñador no busque singularizarse, preocupación que no siempre existe en las obras de restauración. Lógicamente Dezallier, por su época, no atiende al posible carácter de "monumento" del viejo jardín.

Habitualmente se ha procedido trasplantando más o menos mecánicamente al jardín las teorías, normas y procedimientos utilizados en la restauración de edificios, en la convicción de que nada reclama para el 


\section{Defender para la Restauración de Jardines un estatuo autónomo de la Restauración de los demás Bienes Culturales ha sido la habitual justificación de prácticas lesivas para el documento-jardín.}

Este artículo, analizando lo que es específico de los jardines, defiende para ellos, la validez de los criterios generales de la Restauración. jardín una teoría separada de la restauración en general, precisando siempre el distinto tiempo de los procesos (Dezzi Bardeschi, 1992; p. 12). Estando radicalmente de acuerdo con ese punto de partida, es preciso señalar el jardín es un producto estético tan diferenciado del resto de las artes que su restauración presenta especificidades que la separan mucho del comportamiento de las demás. Repasaremos aquí algunas de esas diferencias.

La primera en importancia es la consideración propia del jardín como obra de arte. Su consideración como arte de la naturaleza está implícita (o explícita) en la mayoría de los acercamientos teóricos. Para Assunto

Jardín es, en efecto, la Naturaleza en cuanto, según la ha modelado el hombre para expresar en ella su espíritu (Assunto, 1991; p. 39).

....arte de la naturaleza y naturaleza del arte... (Assunto, 1991; p. 41).

La definición es en sí una paradoja. Naturaleza es lo que no es artificial, y el arte es artificio, producto humano, no-naturaleza. La misma contradicción se produce si aceptamos la base teórica de la restauración según Cesari Brandi (1988; p. 16.), su conocido primer axioma:

\section{Se restaura sólo la materia de la obra de arte.}

La separación entre materia e imagen no puede establecerse de igual forma en las artes plásticas sobre materia inerte y un arte, el jardín, en el que la materia tiene vida propia y en el que la imagen no se debe tanto al artificio humano como al compromiso entre la actividad del hombre y el natural desenvolvimiento de la materia viva. Lo que podría afirmarse incluso para los únicos jardines de materia inerte, los jardines de arena japoneses de la tradición zen, en los que es necesario un "peinado" diario para mantener la forma.

En el jardín, en muchísima más medida que en arquitectura, la forma prístina es una ficción teórica. Mientras que en los edificios suele existir un primer día, un momento inaugural al que poder remitir el valor del origen, en los jardines el proceso de plantación no acaba en una forma definitiva sino que es un paso más en la transición hacia su madurez, situación que se alcanza tras un proceso ininterrumpido de cuidados que son, realmente y no como metáforas, al mismo tiempo mantenimiento y restauración. En los casos más favorables a la teoría de la forma prístina se produce el paso por una serie de estados diversos, regidos por el azar o por procesos naturales y artificiales que el proyectista no controla totalmente. La misma madurez del jardín comparte ese carácter cambiante y en gran medida azaroso, equilibrio dinámico entre permanencia y alteración. Con exclusión quizá de aquellos jardines en que el vegetal está muy controlado y se le demanda una colaboración arquitectónica nítida y estática, podemos afirmar que no suele existir en el jardín el equivalente a lo que en arquitectura podía definirse como forma prístina.

Por otra parte, en el jardín esa imagen viva, móvil y en gran medida imprevisible, no ha sido totalmente proyectada. Como recuerda Scazzosi (1992; p. 20), apoyada en la cita de un clásico de la jardinería italiana, Dell'arte dei giardini inglesi de E. Silva,

L'architetto può scrivere i suoi progetti con chiarezza e comunicarli con precisione; [...] ma l'artista giardiniere [...] rinunci al pensiero di poter rettamente comunicare le sue idee [...] egli dovrà soprattutto disegnare ogni cosa al naturale sul sito.

La praxis proyectual y constructiva en arquitectura y jardinería han sido distintas, también las relaciones entre el proyecto, la dirección de obra y el mantenimiento futuro.

Desde Viollet, gran parte de los restauradores se ha movido por la voluntad de devolver a las obras esa forma prístina que, en los autores más inteligentes, como es, notablemente, el caso del mismo Viollet, estaba desligada de la noción de origen:

[...] obtener su forma prístina, incluso aunque nunca hubiera sido as? 
Son conocidos los avatares que ha sufrido en el debate restaurador esa voluntad. En el caso del jardín ha sido constante su uso y aparece destacado en la Carta de los jardines históricos, conocida como Carta de Florencia, de mayo de 1981, elaborada por el Comité Internacional de los Jardines y Sitios Históricos del ICOMOS-IFLA3. Se recomienda allí el repristino en jardines históricos, se dan incluso las normas documentales y de intervención para asegurar el "carácter científico" del repristino y aconseja que sea ese procedimiento el que se utilice en la cercanía de los edificios. Todo el documento parte de la diferenciación entre dos posibles modalidades de intervención en jardines históricos, la restauración y el repristino. Aunque nunca se dice la diferencia entre uno y otro, se desprende que el segundo es en cierto modo el óptimo al que hay que aspirar aunque las dificultades de conseguirlo hacen permisible que la intervención se quede en "simple" restauro.

El texto fue criticado en Italia donde de forma oficial el Comitato per lo studio e la conservacione dei giardini storici, instituto oficial del Ministero per i Beni culturali e ambientali, propuso la supresión de repristino en la Carta y en concreto de los artículos 9, 15 y 16 que recogían las recomendaciones antes citadas.

Muy diferente a la concepción basada en el concepto de repristino de la Carta de Florencia es la Carta italiana dei giardini storici de septiembre de 198| fruto de las sesiones de grupos de expertos en el tema de la restauración del jardín. El documento, carente de "valor legal", resumía perfectamente la postura de los restauradores que no admitían la utilidad y/o la existencia de la forma prístina. La matriz que generaba su opción en la restauración de jardines era considerar que l'intervento di restauro dovrà rispettare il complessivo processo storico del giardino, lo que se traducía en el rechazo a privilegiare una singola fase assunta in un certo periodo storico (Catalano y Panzini, 1990; pp. 109. I l 0.). No dejaba de tener esta articulación un carácter militante por cuanto la forma prístina de la Carta de Florencia, esa que se consideraba en la historiografía restauradora la generadora de la obra y la única forma realmente válida, se ve reducida aquí, en la Carta de septiembre, a ser una más de las fases de la historia del jardín 4

Poco eco tuvo esta polémica en España. Las restauraciones de jardín han recurrido al término repristino con frecuencia y entre los profesionales mantiene todavía el prestigio de lo colectivamente aceptado. La tradición restauradora de jardines en España es mucho menor que en Italia y eso se traduce no sólo en el menor número de intervenciones de restauración sino también en la menor presencia de artículos teóricos y debates sobre el tema. Ello explica que incluso estudios específicos sobre la Carta de Florencia, como los que realizó Consuelo M. Correcher (1990), no incorporen ningún problema derivado de las repetidas recomendaciones de repristino presentes en el documento, asunto que nosotros consideramos el punto nodal de los errores del texto y que generó todos los debates de interés sobre los problemas la Carta.
Coincidentes con la posición de la Carta italiana dei giardini storici, el problema del repristino sólo nos parece discutible en aquellos jardines cuya pretensión original era la inmutabilidad. Los grandes parterres barrocos eran un artilugio en que el tiempo no existía como elemento del proyecto. Como recogimos en palabras de Dezallier d'Argenville, no hay dificultad en ejecutar los parterres, el problema es mantenerlos como el primer día, con los dibujos de boj limpios, las tierras sin raíces, las arenas de color $\sin$ mezclar, los céspedes perfectos.

Las restituciones realizadas en Het Loo en Apeldoorn (entre 1976-84)5 , en Versalles (diversas fechas, desde el siglo XVIII a la actualidad) ${ }^{6}$ o en el parterre del Privy Garden en Hampton Court (a partir de 1993) 7 responderían a esa voluntad de devolver a su origen un espacio de jardín pensado originalmente como inmutable. En estos casos se trataba de jardines cuya imagen en el momento de la intervención era debida a un proceso evolutivo del que había resultado un cambio de estilo que hacía imposible la "lectura" de una forma anterior, la correspondiente al Barroco. Esa lectura, desde la opción crítica de los restauradores, aparecía como privilegiada y su rescate, en algún caso (Privy Garden), no en todos, se hace eliminando un auténtico jardín antiguo para sustituirlo por una copia discretamente aceptable de una forma perdida de ese mismo jardín.

Aún más, pensamos que la opción se concreta con serios problemas, incluso aceptando la estricta óptica del restaurador "en repristino". En todos los casos el jardín conoció muy diversas formas con pretensiones de inmutabilidad. El restaurador, tras conocer la documentación y tras un estudio arqueológico de los terrenos de las plantaciones, elige una de ellas que resulta privilegiada y en su función se diseña el nuevo jardín. La realidad de Versalles y Hampton Court, que precisamente los estudios previos a la restauración nos descubren con mayor profundidad, nos muestra varios pasados perfectamente asumibles como formas originales (originarias, prístinas). De hecho, afortunadamente, la opción elegida en Versalles no responde a una única forma pasada sino a la mezcla de varias de ellas.

La crítica a la forma prístina no queda sólo en la dificultad de descubrirla o en las dudas sobre su existencia, también en la imposibilidad de devolverla al presente. La forma es un compendio en el que entran vegetales, tecnologías, formas de trabajar de los operarios, entorno natural y urbano. Es imposible volver a aportar todos esos ingredientes, lo que se consigue es realizar un fantasma de aquella forma prístina, en el que la foto actual, comparada con la documentación de época, ofrece un buen porcentaje de aproximación. Las prácticas proyectuales de la jardinería del pasado nos impiden conocer incluso las variedades vegetales que intervenían en la fabricación de la forma. A veces ya no existen, aunque en ocasiones el esfuerzo restaurador ha tratado con interés recuperarlas. La norma habitual en restauración de jardines suele pasar lamentablemente por alto estos aspectos botánicos. 
Junto a las dificultades teóricas y prácticas para restablecer una forma prístina tienen para nosotros absoluta validez las consideraciones derivadas de la afirmación de Cesare Brandi (1988, p. 33):

El único momento legítimo que se ofrece para la acción de la restauración es el presente mismo de la conciencia observadora. [...] La restauración, para presentar una operación legítima, no deberá concebir el tiempo como algo reversible, ni la abolición de la historia.

En el jardín, como obra de arte y como documento, tienen valor las huellas del tiempo, el equivalente a la pátina de las artes plásticas. La restauración del jardín, que tiene por objeto permitirnos la lectura de su unidad, respetando el término del mismo Brandi (1988, p. 23), sólo debe considerar problemáticas las intervenciones del pasado que hayan perjudicado esa lectura.

Otra diferencia notable del jardín es su carácter polimatérico, además de vegetales y edificaciones, entran en su composición caminos, adornos mobiliarios y, con notable importancia, elementos ajenos a los límites del jardín como son los del paisaje que intervinieron en el proceso mismo de su diseño. La vista desde un belvedere pertenece al propio jardín, aunque la mayoría de las veces su conservación o recuperación exceda los límites operativos del proceso de restauración del jardín.

Las distintas materias que componen el jardín tienen también un diferente ritmo de crecimiento, deterioro y muerte. Afirmación que es válida así mismo para el conjunto de los componentes vegetales. El proceso de conservación de un jardín debe atender a esos diferentes ritmos y ello determina un programa de cuidados en el que se contemplan ciclos de atención diarios, semanales, y así sucesivamente hasta ciclos muy largos donde los cuidados a los elementos vegetales "más lentos" del jardín, los grandes árboles, deben estar previstos.

La materia vegetal del jardín además de sus ritmos singularizados es también una materia plástica que permite su utilización, y por tanto su permanencia durante procesos de cambio de estilo o de forma en el jardín. Esta característica es singularmente importante pues permite variadas operaciones al estudioso del jardín y al restaurador. La permanencia de elementos de anteriores fases ayuda a descubrir y descifrar como fueron. Para el restaurador, contar con la presencia de esos elementos vegetales, plásticos, le permite operar sobre la misma materia original del jardín para restaurar las formas.

Un paseo de tilos que hoy aparecen como árboles tallados en bola más o menos informal, pudo ser en el siglo pasado una pantalla en alto podada "a la francesa". Un círculo de cipreses en un jardín granadino pudo ser hace cien años una glorieta de cipreses tallados. La plasticidad de los vegetales no encuentra parangón en la arquitectura donde los nuevos estilos y los cambios de forma operan de manera distinta, lo que en arquitectura es excepción, aprovechar viejos materiales en cambios de forma, en el jardín es la norma.

La plasticidad del verde implica también un debate sobre los términos copia, original, fraude o falso histórico. Volver a podar los tilos de un paseo tal y como se podaron en el siglo XIX esos mismos pies de árbol, es un acto que desafía las simplificaciones teóricas. La forma actual se debe al abandono del tallado, seguramente debido al cambio de gustos/propietario y a motivos económicos, pero es una forma consolidada como "la propia" del jardín. Recuperar el viejo tallado puede ser un ejercicio más cercano a una anastilosis que a un repristino. Volver a tallar una glorieta de ciprés o colocar los cipreses perdidos, ies realizar una copia?. Acaso no son ambos auténticos, el elemento y la forma: un ciprés es, en cualquier caso, un ciprés, no la copia de un ciprés8.

Lo dicho hasta aquí apunta también la importancia de los aspectos tecnológicos en el mantenimientorestauración del jardín. El mantenimiento del jardín, lo hemos visto, determina permanentemente su forma. No sólo las operaciones directas de los jardineros sobre la materia verde. Nuestro estudio sobre los cármenes granadinos detalla como la estructura de los cármenes depende del sistema tradicional de riego (Cf. Tito Rojo, 1997; Tito Rojo y Casares Porcel, 1999). Hemos comprobado que el cambio de ese sistema, sustituyendo la inundación a manta de los cuadros por sistemas de goteo o aspersión, se está produciendo en muchos cármenes granadinos. En nuestra opinión la inclusión de esas nuevas tecnologías, aparte de su falta de idoneidad, hace que la estructura del jardín, su estética, ya no esté en consonancia con la forma de regar. Esa contradicción significará que paratas y cuadros sean durante un breve tiempo restos de una antigua forma conservados por tradición y no por su utilidad. Inevitablemente desaparecerán dentro de poco en beneficio de nuevas formas coherentes con la tecnología de mantenimiento. Ya en muchos cármenes hay céspedes, allí donde a principios del siglo XX, por similares razones, jardines ornamentales habían ocupado el lugar de antiguos huertos.

\section{El jardín como palimpsesto y opera aperta}

Partendo dunque dalla realtá d'oggi per cui espesso il giardino nel corso dei secoli ha subíto varie sovrapposizioni e metamorfosi vegetali, ne possiamo dedurre che occorre guardarlo e studiarlo quasi come un palinsesto vegetale.

Con estas palabras introducía Pasolini Dall'Onda (1975; p. 33) en la teoría de la restauración de jardines la feliz metáfora del palimpsesto, tras él la reutilizan aquellos que han pretendido no devolver al jardín los elementos de la "antica armonia originaria" en el esfuerzo de un repristino imposible, sino respetar la "attuale nuova fusione"". 
La imagen del palimpsesto servía a los efectos de delatar que, en el jardín histórico, en el mismo lugar y en los mismos vegetales se encuentran rastros de trazas, intervenciones puntuales, transformaciones de diversas épocas, incorporaciones de pies vegetales, aportes de nuevos catálogos florísticos. Da ello al documento jardín un valor testimonial importante sobre el pasado del propio jardín y de las jardinerías de sus pasados. Muy gráficamente Scazzosi (1992; p. 27) profundiza en la imagen del pergamino reutilizado usando para el jardín verbos pertenecientes al mundo de la escritura, así el documento jardín, palimpsesto vegetal, ha sido escrito, reescrito, modificado, corregido, anotado, cancelado, continuamente reutilizado.

Esta misma autora aportó otro modelo útil a la hora de establecer criterios de restauración para los jardines. El carácter inacabado del jardín, la imposibilidad de referir para la mayoría de ellos un tiempo (pasado, presente o futuro) que pueda ser considerado la forma de la obra, ante la cual las formas previas son su gestación y las posteriores su permanencia o su deterioro, dio pie a Lionella Scazzosi para hablar del jardín como obra abierta. En un doble sentido, en tanto la arquitectura vegetal está inscrita en un recorrido temporal que implica una serie de presentes en los cuales se escoge siempre un camino, entre los que se encuentran incluso los del abandono, la destrucción o la detención ilusoria en un estado determinado, y en tanto que el proceso de mantenimiento significa la elección de un equilibrio entre permanencia e innovación que genera en cada presente una diferente autenticidad.

El concepto obra abierta remite al fijado por Umberto Eco en Opera aperta, lo que puede dar lugar a algún equívoco, en tanto se utiliza aquí de forma diferente. En Eco se establecía como una relación entre producción-obra-fruición; en ella, la obra es el punto de llegada de una producción y el punto de partida de consumos que al realizarse dan vida, siempre desde perspectivas diferentes, a la forma inicial (Eco, 1979; p. 40.). Es distinto su uso referido a una obra permanentemente inacabada, o mejor aún, a una obra permanentemente acabada de forma distinta, como es el jardín.

Eco hablaba del modelo de obra abierta como «un modelo absolutamente teórico e independiente de la existencia factual de obras definidas como "abiertas"») (Eco, 1979; p. 42). Consideración que permitió al autor ironizar sobre las pretensiones de algunos artistas de realizar "obras abiertas" tras el éxito de las primeras ediciones de su libro. La estructura de obra abierta era para Eco el modelo común de las obras contemporáneas que «sugieren la imagen de un mundo ambiguo y pululante de determinaciones posibles» (Eco, 1979; p. 55); usar esa imagen para la obra-de-arte-jardín, no para determinadas obras-de-arte-(jardín)-contemporáneas, se hace forzando el sentido de los textos de Eco. Dicho esto la noción de obra abierta puede ser muy útil para fijar el comportamiento del fenómeno obrade-arte-jardín y para optar en consecuencia por un modelo restaurador que huya de la ficción teórica de la forma prístina, o al menos, para fijar una determinada actitud ante la restauración del jardín.
La peculiaridad del jardín como obra permite considerarla abierta en numerosos sentidos. En otro lugar hemos apuntado la similitud del jardín con la música ( Tito Rojo, 1995; p. 16) en tanto que, obra que se desarrolla en el tiempo, el jardín es la suma de ciclos vitales de las plantas, y en tanto que el jardín, como la música, se interpreta. Es afortunada la coincidencia con el hecho de que la elaboración de la Opera aperta por Eco partiera de la lectura de varias obras musicales, en las que, al sentido normal de tener que ser interpretadas a partir de la forma elaborada por el autor, se unía que esa forma estaba organizada como una serie de posibilidades cuya elección se confiaba al intérprete (Eco, 1979; p. 74.). También en ese sentido el jardín es obra abierta, pues la práctica proyectual del jardín confía gran parte de la opción de forma definitiva a la interpretación de los ejecutantes. Aunque hay que decir que siempre se ha partido de la confianza en que el resultado de la plantación solía estar determinado por las costumbres del momento y, aún sin estar especificado tan exactamente como en la praxis proyectual de las construcciones, el aspecto final del jardín se consideraba siempre dentro de lo previsible.

De estas consideraciones se deduce que en el jardín no es posible establecer el debate entre restauración y conservación en los mismos términos que en las artes plásticas, incluida la arquitectura. En la mayoría de los jardines, aquellos en que los vegetales no cumplen una función estrictamente geométrica, el dilema es falso: conservar es restaurar. La única alternativa a esa conservación restauradora sería el abandono. La conservación que pedía Ruskin para los edificios, que alargara la vida y a la vez respetara su muerte digna, no es posible en el jardín so pena de considerarlo un arte, más que efímero, fugaz. En él la conservación interviene, y lo hace de forma tan global e intensa que no puede compararse a las conservaciones de los edificios.

A pesar de ello solemos usar, también en el jardín, de forma diferenciada los términos restauración y conservación. La conservación debe operar sobre la obra de jardinería sin alterar su lectura. La restauración pretende precisamente cambiar la lectura, para hacerla más correcta.

El jardín histórico tiene valores estéticos y acumula información que nos habla del pasado, político, económico, agrícola, botánico, urbano. Es a la vez una obra de arte y un documento. El deterioro del jardín lesiona la lectura de ambos aspectos. El objetivo de la restauración es, en el aspecto estético, hacer más clara la singularidad del jardín, lo que hace de él obra de arte, sin lesionar el valor de su aspecto documental, el entramado de datos que el paso del tiempo en el lugar le ha dejado inscrito. Para ello es imprescindible que la restauración del jardín realice una operación previa de análisis que desvele toda la información que almacena el jardín, de otra forma el proceso restaurador puede, y ocurre en la mayoría de los casos, eliminar informaciones valiosas del palimpsesto. Esa operación diagnóstica y las opciones que de ella se deducen serán diferentes en cada caso, pero en nuestra experiencia son los elementos vivos, los vegetales, la mayor fuente de información 10 . 


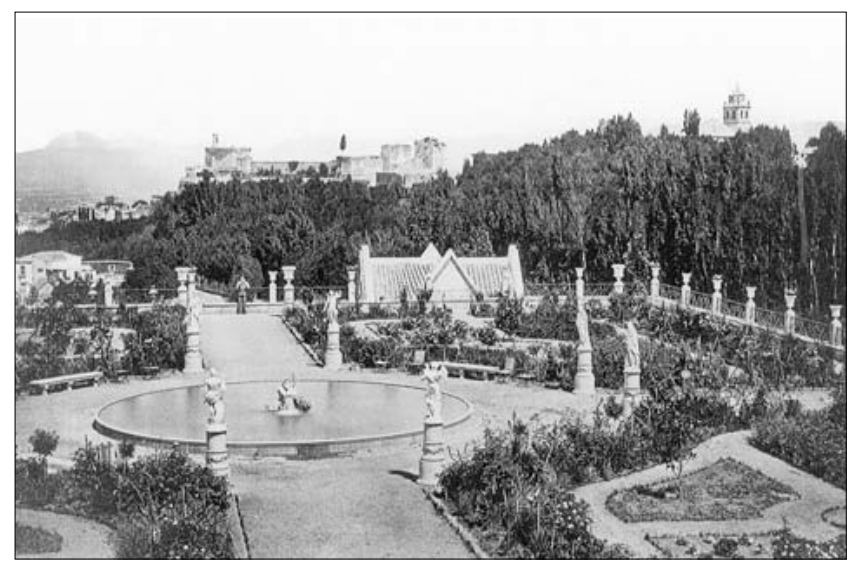

EJEMPLO DE CONFIGURACIÓN DE UN "PALIMPSESTO"

EN UN JARDÍN HISTÓRICO:

EL JARDÍN FRANCÉS DEL CARMEN DE

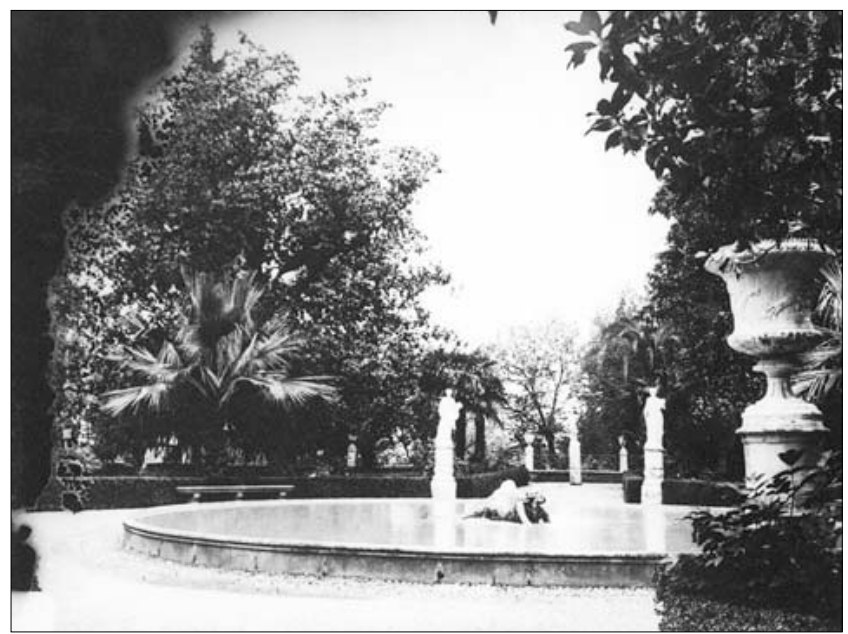
LOS MÁRTIRES (GRANADA)

Imagen I ${ }^{\text {a. }}$ : El Jardín Francés recién construido. Fotografía de Charles Clifford de I861

Imagen 2a: El mismo jardín hacia 1920 Los magnolios, que se veían muy pequeños en la imagen $I^{a}$, son ya grandes árboles, las estatuas han cambiado de sitio, y se han introducido nuevos elementos, tanto vivos (las palmeras) como inertes (la ninfa del estanque que sustituye al simbólico Río de |86|).

Imagen $3^{\text {a }}$ : Palimpsesto actual, en él se mezclan:

elementos originales (los setos, las estatuas y la estructura del jardín),

elementos originales transformados (los

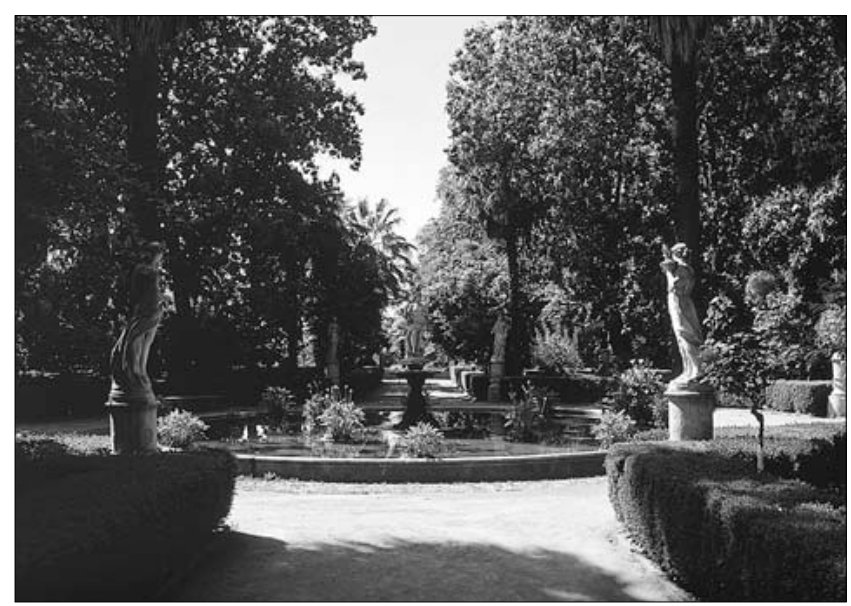
magnolios de $|86|$ y los palmeras de principios del siglo XX han crecido, las estatuas han cambiado de nuevo de sitio -significativamente en el centro del estanque está colocada la estatua de Neptuno que en |86। estaba en el círculo exterior-) y elementos nuevos incorporados (los arbustos de flor de los cuadros, los naranjos y más palmeras).

El paso del tiempo, más que una deliberada intervención transformadora, ha acabado convirtiendo un "versallesco" jardín original, con gran estanque central rodeado de estatuas y bordados de flor entre setos de boje, en un umbrío jardín "a la granadina", con parterres de grandes árboles y arbustos de flor sujetos pro altos setos.

\section{El criterio de la restauración}

En palabras de Capitel (1988; p. 49), "entre el mimetismo historicista o el clacisismo de manual, y el collage moderno, hay todavía un ancho campo que explorar". Existe hoy una fuerte tendencia a plantear la restauración como un problema de diálogo con el bien a restaurar, en el que, sin reglas fijas, estudiándolo y apre(he)ndiendo su código particular, el restaurador determina qué hacer. Parte este criterio de la convicción de que la restauración es un hecho irrepetible y único, tan singular como para desafiar la aplicación estricta de un sistema de actuaciones y prohibiciones preestablecido 'I. Esta postura es, más o menos matizada, la predominante en el ámbito italiano, y desde aquí asimilada por los teóricos de la restauración españoles. Se convierte de alguna manera en la postura de más consenso, sin que ello signifique que todos los que se hacen eco de ella tengan posturas coincidentes, ni mucho menos sus prácticas ya que, como denuncia Martínez Justicia, existe en el campo de la restauración arquitectónica una "total dicotomía entre la 
teoría y la práctica" (1992; p. 582), existe una contradicción entre memorias y declaraciones de principios ajustadas a modelos teóricos prestigiados y prácticas sujetas a un "sentido común" reconstructor.

Se deduce de aquí que el criterio de la restauración no puede establecerse según un código de reglas fijas, ya sea con el mandato del repristino, o con el imperativo de un catálogo de prohibiciones, no reconstruirás, no eliminarás, no reintegrarás...

El problema de la actuación en jardinería es que, desde nuestro punto de vista, tanto desde la teoría como desde la práctica, la restauración es inevitable. En el viejo dilema restaurar-conservar, aplicado al jardín, optar por la conservación pensando que no conlleva implícita el equivalente a una restauración es ignorar como funciona el jardín. De igual manera, ante ese dilema, restaurar ignorando que el objetivo primordial es conservar el jardín sobre el que se trabaja, es comportarse como los "vándalos" que denunciara Ruskin'2.

Queda pues claro para nosotros que el proyecto de restauración de un jardín debe tener en cuenta ante todo la consideración del jardín como documento; un texto complejo y heterogéneo ("polimatérico") que se presenta como un palimpsesto. El interés del proyecto, desde este punto de vista, no debe ser recuperar un momento original desde donde poder dictaminar el come era e dove era objetivo de todo repristino, sino constituir un nuevo conjunto unitario con valor propio, un nuevo jardín, que respete meticulosamente las informaciones acumuladas y conservadas en el documento y las lecturas inherentes a su existencia. Siempre que esto sea posible el resultado será la constitución de un jardín que, siendo en gran medida el objeto de valor histórico y estético que se consideró que valía la pena recuperar-restaurar-reinstaurar-salvaguardar es al mismo tiempo testimonio de la historia de ese lugar, del paso del tiempo acumulado en ese jardín.

Pudiera parecer que la tendencia que señalamos de fijar la atención, más que en las reglas, en el caso particular, libera al restaurador de un compromiso previo con una "tecnología" restauradora. No es así, muy por el contrario sólo el conocimiento de lo que denominamos cultura de la restauración, su historia, su técnica, sus tecnologías, permite al responsable de una restauración operar con criterio.

Esto significa que, de la misma forma que para conocer el jardín que se quiere restaurar hay que saber de jardines, para restaurar el jardín hay que saber de restauración de jardines. La restauración es ya una cultura y no puede someterse la de un jardín histórico a decisiones tomadas sin estar en conocimiento de las formas de funcionar y pensar en ese tipo de actividad. El carácter pionero que tiene la restauración de jardines no excusa, sino que aumenta, la necesidad de que los responsables de una restauración tengan los elementos teóricos, los criterios y herramientas, necesarios para abordarla. Como se manifestó en los II Encuentros en la Alhambra, existe

...la necesidad de vincular la restauración al conocimiento histórico, artístico, historiográfico, científico y de la propia cultura restauradora... 13

Lamentablemente, jcuantos jardines históricos han sido restaurados por personas que ni saben de jardines, ni saben de restauración de jardines!. Lamentablemente este es el punto y final de esta reflexión pero en las prácticas lo que conocemos es todavía un punto y seguido.

\section{Notas}

I. Dezallier d'Argenville, 1972; p. 16. La edición consultada es la facsímil de la cuarta edición de 1747 en impresión de 1760 ignoramos si el texto aparecía ya en las anteriores, I $^{\text {a }}$ de 1709, $2^{\text {a }}$ de 1713, $3^{\text {a }}$ de 1722). Sobre las ediciones de esta obra consúltese la introducción de la facsímil y Ganay, 1989.

2. Viollet-le-Duc, 1858, voz "Restauration".

3. El texto de la Carta de Florencia ha sido publicado en varias ocasiones, no siempre de forma correcta (es habitual, por ejemplo, el error de traducir el francés "essences" por "esencias", cuando en realidad se trata de "especies vegetales") Correcta es la traducción incluida en Añón, Luengo y Luengo (1995, pp. 403-406), en ella se ha optado por traducir "repristino" por "restitución", atendiendo a la versión francesa de la Carta.

4. El debate creado por los oponentes a la Carta de Florencia aparece recogido en numerosos trabajos, puede consultarse sobre él a Belli Barsali (1981, 1993), Catalano y Panzini (1990; pp. | | |-| |3) y Dezzi Bardeschi (|99|; pp. 304-3 |5).

5. Cf. Asbeck, 1990 y Catalano y Panzini, 1990; pp. 23-27 y II4. En este último se incluye una abundante bibliografía sobre la restauración de Het Loo.

6. Lablaude, 1992; Taylor le Duc, 1994.

7. Thurley, 1995; Jacques, 1995; Batey y Woudstra, 1995; Wright, 1998;
8. Habría que añadir, un ciprés de la misma variedad, edad (?)... E tema de original y copia en la restauración del jardín es un tema apasionante que no podemos abordar aquí y que afecta a una parte importante de la teoría de la restauración de jardines. En el desarrollo de nuestro discurso hemos tenido presente una frase de L. Scazzosi, 1992; p. 27: "in tal modo va in crisi ogni tradizionale concetto di autenticità e di copia dell'opera".

9. Cf. Pasolini Dall'Onda, 1975; Catalano y Panzini, 1990; Boriani, 1992; Scazzosi, 1992. El término aparece también en autores ingleses, Mitchell; 1985 y es recogido en la Carta italiana dei giardini storici de Florencia, septiembre 1987.

10. Cf. como ejemplo nuestro análisis del Jardín Español del Carmen de los Mártires (Tito Rojo, 1997; pp. 4.109-4-1 16 y 4.190-4.192)

II. Cf. Boriani, 1992; Capitel, 1988; Catalano y Panzini, 1990; Scazzosi, 1992; Martínez Justicia, 1992.

12. "Turba" en la edición española, Ruskin, 1987, p. 184. Dezzi Bardeschi, 1991, p. 72, recogiendo la denuncia de Ruskin utiliza "vandali".

13. Organizados en noviembre de 1989 por el Patronato de la Alhambra, el Colegio Oficial de Arquitectos de Andalucía Oriental y la Scuola di Specializzazione per lo Studio ed il Restauro dei Monumenti, bajo el título genérico de Conocimiento y Restauración, Cf. Martínez Justicia, 1992; p. 581 


\section{Bibliografía}

AÑÓN FELIU, CARMEN. 1991. "The Restauration of the King's Garden at Aranjuez", en The Autentic Garden, edt. Clusius Foundation, Leiden, pp. 97-103.

AÑÓN, CARMEN. 1992. "Alameda de Osuna. El Capricho: il Labirinto", en Il giardino e il tempo, Edt. Guerini et Associati, Milano, pp. | $25-132$.

AÑÓN FELIÚ, CARMEN. 1993. "El jardín histórico: Notas para una metodología previa al proyecto de recuperación", en Jardins et sites historiques, Edt. Icomos-Unesco. Madrid, pp. 312-325.

AÑÓN, CARMEN; LUENGO, MÓNICA y LUENGO, ANA. 1995. Jardines artísticos de España. Edt. Espasa Calpe. Madrid.

ASBECK, J. VAN. 1990. "Il giardino formale di Het Loo", en L'architettura dei giardini d'Occidente al Rinascimento al Novecento, edt. Electa, Milano, pp. I74-I76.

ASSUNTO, ROSARIO. 1991. Ontología y teleología del jardín. Edt. Tecnos. Madrid.

BATEY, MAVIS y WOUSTRA, JAN. 1995. The story of the Privy Garden at Hampton Court. Edt. Barn Elms Publishing. Glasgow.

BELLI BARSALI, I. 1981. "II Giardino storico italiano: Problemi di indagine", en Il giardino storico italiano. Problemi di indagine, fonti letterarie e storiche, Firenze, Actas del Convegno di studi, Siena-San Quirino d'Orcia, 6-8 ottobre, 1978, edt. Leo S. Olschki, pp. 19-29.

BELLI BARSALI, I. 1993. "Per una carta del restauro dei giardini storici", en Jardins et sites historiques, edt. ICOMOS \& Doce Calles, Madrid, pp. |82-186.

BRANDI, CESARE. 1988. Teoría de la restauración. Edt. Alianza Editorial. Madrid.

BORIANI, M. 1992. "Quale restauro per le «architetture vegetalis)? Problema di tutela, conservacione e gestione di un patrimonio viviente" en II giardino e il tempo. Conservacione e manutenzione delle architetture vegetali. edt. Guerini E Associati, Milano, pp. 15-24.

CAPITEL, A. 1988. Metamorfosis de monumentos y teorías de la restauración. Edt. Alianza Editorial. Madrid.

CASARES PORCEL, MANUEL y TITO ROJO, JOSÉ. 1998. Metodología de la restauracion en jardinería histórica. Departamento de Diseño para el Medio Ambiente. Universidad Autónoma de México, Azcapozalco.

CATALANO, M. \& F. PANZINI. 1990. Giardini storici. Teoria e tecniche di conservazione e restauro. Edt. Officina edizioni. Roma.

CORRECHER y GIL, C.M. 1990. "De los jardines Históricos: Los criterios de restauración y sus normas", Zona verde, 26:29-34

DEZALLIER D'ARGENVILLE, A.J. I 972. La théorie et la practique du jardinage, mit einer einleitung von Hans Foramitti. Edt. Georg Olms Verlag. New York [edición facsímil de la $4^{a}$ edición parisina de 1760].

DEZZI BARDESCHI, M. 199I. "La Carta italiana dei giardini storici otto anni dopo" en Dezzi Bardeschi, M. 1991. Restauro: punto e da capo. Frammenti per una (impossibile) teoria, edt Franco Angeli, Milano, pp. 304-3I5.

DEZZI BARDESCHI, M. 1992. "Quei giardini fuggitivi come gli anni..." en Il giardino e il tempo. Conservacione e manutenzione delle architetture vegetali, edt. Guerini E Associati, Milano, pp. | | - | 4 .
ECO, U. 1979. Obra abierta. Edt. Ariel. Barcelona [de acuerdo con la segunda edición italiana de 1967, primera de 1962].

GANAY, E. DE. 1989. Bibliographie de l'Art des Jardins (Essai de Bibliographie des ouvrages publiés en français sur l'Art des Jardins). Edt. Union des Arts Dècoratifs. Paris.

ACQUES, D. 1995. "The history of the Privy Garden", Apollo, I 42(403):23-42.

LABRAUDE, P.A. 1992. "Il Parco di Versailles: progetti e realizazioni" en Il giardino e il tempo. Conservacione e manutenzione delle architetture vegetali, edt. Guerini E Associati, Milano, pp. |15-124.

MARTÍNEZ JUSTICIA, M. J. 1992. "La restauración arquitectónica y sus formulaciones teóricas. A propósito de la restauración de la fachada de la Real Chancillería y de la portada de la Casa de Castril", Cuadernos de Arte de la Universidad de Granada, 23:581 -596.

MITCHELL, A. 1985. "Regénérer les jardins classiques", Cahiers de la section française de l'lcomos.

PASOLINI DALL'ONDA, D. 1975. "Restauro del verde storico nella pianificazione del territorio", Italia nostra, 128:30-33.

RUSKIN, J. 1987. Las siete lámparas de la arquitectura. Edt. Stylos. Barcelona. [Edición original de I849].

SCAZZOSI, L. 1992. "II giardino opera aperta: il progetto di conservazione e il tempo", in II giardino e il tempo, edt. Guerini E Associati, Milano, pp. 25-58.

TAYLOR LE DUC, S. B. 1994. "Louis XVI Public Gardens. The Replanation of Versailles in the I8th Century", Journal of Garden History, |4(2):67-9|

THURLEY, S. 1995. "William III's Privy Garden at Hampton Court Palace: Research and restoration", Apollo, I42(403):3-22

TITO ROJO, JOSÉ. 1995. Jardines. Edt. Festival Internacional de Música y Danza de Granada. Granada.

TITO ROJO, JOSÉ. 1997. Restauración en Arquitectura del Paisaje. Ensayo metodológico aplicado al Carmen de los Mártires y otros jardines granadinos del siglo XIX. Tesis doctoral. Universidad de Granada.

TITO ROJO, JOSÉ y CASARES PORCEL, MANUEL. 1998. "La restauración del Jardín Botánico de la Universidad de Granada". Parjap. 5:17-21.

TITO ROJO, JOSÉ y CASARES PORCEL, MANUEL. 1999. EI Carmen de la Victoria. Historia de un jardín regionalista. Universidad de Granada. Granada.

VIOLLET-LE-DUC, E. I854-68. Diccionaire raisonné de l'architecture française de XI au XVI siecle. Paris.

WRIGHT, TOM. 1998. "Hampton Court Palace, London; Some Historical Aspects, and the Recent Restoration of the Privy Garden", en Felipe II, el Rey íntimo. Jardín y Naturaleza en el siglo XVI, edt. Sociedad Estatal para la Conmemoración de los Centenarios de Felipe II y Carlos V, Aranjuez, pp. 447-466. 